\title{
In vivo application of a small molecular weight antifungal protein of Penicillium chrysogenum (PAF)
}

\author{
Zoltán Palicz ${ }^{1}$, Ágnes Jenes ${ }^{1}$, Márta Füzi ${ }^{1}$, Kornél Miszti-Blasius ${ }^{2}$, Csaba Hegedüs ${ }^{3}$, \\ László Virág ${ }^{3}$, Sándor Kollár ${ }^{4}$, Ilona Kovács ${ }^{4}$, Miklós Emri ${ }^{5}$, Teréz Márián ${ }^{5}$, Éva \\ Leiter $^{6}$, István Pócsi ${ }^{6}$, Éva Csősz ${ }^{7}$, Gergő Kalló $^{7}$, László Csernoch $^{1}$ and Péter Szentesi $^{{ }^{*}}$ \\ ${ }^{1}$ Department of Physiology, Medical and Health Science Centre, University of Debrecen, \\ Debrecen, Hungary
}

${ }^{2}$ Department of Clinical Biochemistry and Molecular Pathology, Medical and Health Science Centre, University of Debrecen, Debrecen, Hungary

${ }^{3}$ Department of Medical Chemistry, Medical and Health Science Centre, University of Debrecen, Debrecen, Hungary

${ }^{4}$ Department of Pathology, Kenézy Hospital LTD, Debrecen, Hungary

${ }^{5}$ Department of Nuclear Medicine, Medical and Health Science Centre, University of Debrecen, Debrecen, Hungary

${ }^{6}$ Department of Microbial Biotechnology and Cell Biology, Faculty of Science and Technology, Centre of Arts, Humanities and Sciences, University of Debrecen, Debrecen, Hungary

${ }^{7}$ Proteomics Core Facility, Department of Biochemistry and Molecular Biology, Medical and Health Science Centre, University of Debrecen, Debrecen, Hungary

Running title: In vivo application of PAF 
* - Author for correspondence: Dr. Péter Szentesi, Department of Physiology, Faculty of Medicine, Medical and Health Science Centre, University of Debrecen, P.O.Box 22, H-4012 Debrecen, Hungary; Tel: 36-52-255575, Fax: 36-52-255116, E-mail: szp@phys.dote.hu 


\section{Abstract}

The small antifungal protein secreted by Penicillium chrysogenum (PAF) inhibits the growth of important zoo pathogenic filamentous fungi, including members of the Aspergillus family. It was shown previously that PAF has no toxic effects on mammalian cells in vitro. We carried out safety experiments by investigating the in vivo effects of PAF by inoculating adult C57/B16 mice with PAF intranasally. Animals were randomly divided into six groups and subjected to different PAF concentrations, up to $54 \mu \mathrm{g}$, either once a week for up to 8 weeks or once every day for two weeks. Animals neither died due to the treatment nor were any side effects observed. Histological examinations did not find any pathological reaction in the liver, in the mucous membrane of the nose, and in the lungs, even in the highest concentration used. Mass spectrometry revealed that ZZZ. The effect of the drug on the skin was examined in an irritative dermatitis model by measuring the thickness of the ears. This proved to be the same following PAF application as in control $(23.8 \pm 9.2 v s .22 .5 \pm 5.0 \mu \mathrm{m}$, respectively) and significantly less than when treated with phorbol-12-myristate-13-acetate (PMA; $57.5 \pm 29.2 \mu \mathrm{m}$ ) used as positive control. Histological changes relative to control were present only in the case of PMA. Positron emission tomography was used to follow potential inflammation of the lungs. Neither the application of control saline nor that of PAF induced any inflammation while the positive control lipopolysaccharide did. Since no toxic effects of PAF were found either in intranasal application or in local external treatment, our result is the first step for introducing PAF as potential antifungal drug in human therapy.

Key words: antifungal protein, aspergillosis, PET, dermatitis, histology 


\section{Introduction}

Aspergillosis is one of the most severe human diseases caused by fungi threatening especially immunodeficient patients. The condition caused by various pathogenic Aspergillus species affects particularly the lungs. The most important species in this respect is Aspergillus fumigatus which preferentially colonizes caverns caused by previous diseases such as tuberculosis (21). It can cause allergic bronchopulmonary aspergillosis, pulmonary aspergilloma and invasive aspergillosis. In the latter the symptoms can be cough, fever, lowgrade chest pain, dyspnea and hemoptysis (2) while in allergic bronchopulmonary aspergillosis malaise, fever, presence of sputum plugs and purulent sputum, cough, chest pain, and hemoptysis can be observed beside the symptoms of asthma (23). The pulmonary aspergilloma can cause hemoptysis or it can be without symptoms. Other symptoms can be present due to the underlying pulmonary disease (ZZZ). Although a number of antifungal drugs are in use, there is a rising tendency of resistant strains leading to a high mortality rate (7), thus rendering the finding of new antifungal treatments of high interest.

Filamentous fungi produce a wide spectrum of defensive proteins some of which, including the antifungal protein secreted by Penicillium chrysogenum (PAF; 13), are designed to hinder the growth of other fungi. PAF is a low molecular weight, basic, and cysteine-rich protein which has a homology with other antifungal proteins produced by other fungi. PAF inhibits the growth of various pathogen fungi including A. fumigatus, A. nidulans, A. niger, Botrytis cinerea (8). It causes an increased production of reactive oxygen radicals, reduces the metabolism of the fungal cells, and initiates an increased potassium efflux (8). PAF enters the cells via an active transport mechanism (16) and acts intracellularly, probably through heterotrimeric G-protein signaling $(10,14)$. It causes the hyperpolarisation of the membranes of the fungal cells, which leads to the disintegration of the membranes and, consequently, an 
apoptosis-like phenotype appears. Furthermore, reactive oxygen species oxidize intracellular proteins, lipids, and nucleic acids, causing not only the disintegration of the cellular membranes but also that of the mitochondria (20). These toxic effects, when they reach a critical limit, also contribute to the programmed cell death of the affected fungi (6).

In fungal colonies treated by PAF the number of cells showing apoptotic signs including increased phosphatidylserine externalization and DNA fragmentation are increased compared to control colonies while the proportion of the necrotic cells remains the same (10). On the other hand, in a variety of mammalian cells PAF had no toxic effects. The ionic currents of neurons and astrocytes, the potassium and L-type calcium currents of skeletal muscle fibers were not altered. No toxic effects were found on endothelial cells with MTT assay ZZZ and only a minor pro-inflammatory effect was observed on human blood incubated with PAF when the level of cytokines was measured (22).

The glucose analog 2-[ $\left[{ }^{18} \mathrm{~F}\right]$ fluoro-2-deoxy-D-glucose $\left({ }^{18} \mathrm{FDG}\right)$ based positron emission tomography (PET) imaging is a very sensitive modality for the detection of metabolic activity of tissues. ${ }^{18}$ FDG uptake of infectious processes - mainly due to the presence of macrophages - has already been reported (Knight et al. 1996), suggesting that ${ }^{18}$ FDG based PET (FDGPET) can accurately be used for staging mediastinal diseases. The innate host defense system against Aspergillus includes phagocytic cells (peripheral blood monocytes, macrophages, neutrophils, etc.) (Walsh et al. 2005). Several mechanisms have been proposed as the basis for ${ }^{18} \mathrm{FDG}$ uptake into the inflammatory cells but they all conclude that cells accumulate ${ }^{18} \mathrm{FDG}$ with high concentration, depending upon the degree of stimulation, which is a function of inflammatory activity (Basu et al. 2010).

We developed an animal model for exploring the possible effects of PAF in living mice and we used a new non-invasive small animal PET imaging technique to prove that the drug is not toxic. Employing these techniques, this is the first study to indicate that the low 
molecular weight antifungal protein PAF has no toxic effects in vivo. Although further investigations should be carried out in vivo, including safety and efficacy studies, our results support the possible therapeutic application of PAF against aspergillosis in humans.

Part of this work has been presented to the Hungarian Physiological Society (17). 


\section{Materials and Methods}

\section{Purification of PAF}

Purification of PAF was carried out as described previously (22). Briefly, $P$. chrysogenum NCAIM 00237 was grown in a sucrose $(20 \mathrm{~g} / \mathrm{l})-\mathrm{NaNO}_{3}(3 \mathrm{~g} / \mathrm{l})$ minimal medium for $72 \mathrm{~h}$ at $25^{\circ} \mathrm{C}$ with shaking (13). Mycelia were removed with centrifugation and the low molecular weight protein fraction of the supernatant was separated in Amicon Stirred Cells (Millipore, Billerica, MA, USA). PAF was purified with ion exchange chromatography on a CM Sephadex Fast Flow column (Amersham-Pharmacia, Uppsala, Sweden). The quality of the preparation was always checked with SDS-PAGE on pre-cast Novex $16 \%$ Tris/glycine gels (Invitrogen Life Technologies, Carlsbad, CA, USA), where protein bands were visualized with Coomassie Brilliant Blue R staining. PAF was dissolved in physiological saline (ZZZ) for the in vivo experiments.

Model of PAF-induced lung effects

12 weeks old C57/B16 mice of both sexes were used in all in vivo experiments. First the mice were randomized into five groups (a control group and mice treated with $0.1,0.5,2.5$, and $5 \mu \mathrm{g}$ PAF dissolved in physiological saline; 10 animals per group). The control group was treated with drug free physiological saline. Mice were slightly anesthetized with penthobarbital (50 mg/kg, $4 \mathrm{mg} / \mathrm{ml}$ concentration, $250 \mu$ per mouse ZZZ) intraperitoneally then the above solutions were administrated by pipettes into the nose of the animals in a $50 \mu 1$ final volume. Under these conditions the solution reaches the lungs through normal breathing. The efficiency of the procedure was tested by applying a dye solution (see Supplementary Figure). The animals were treated weakly for 8 weeks. An extra group $(n=6)$ was treated with a very high dose of PAF $(54.55 \mu \mathrm{g}, 2727.5 \mu \mathrm{g} / \mathrm{kg} \mathrm{ZZZ)} \mathrm{for} 5$ weeks. In this group blood 
samples were taken before and at the end of the application of the drug. To investigate the acute effect of PAF two groups of mice (10 animals per group) were treated daily for two weeks. The control group was treated with PAF free physiological saline while the protein was administrated in $5 \mu \mathrm{g}$ dose in the other group of animals.

ZZZ To explore the possible side effects of PAF, in both type of experiments the lungs, the kidneys, and the liver of the animals were examined histologically in each group. ZZZ kell ez itt?

Model of LPS-induced acute lung inflammation

For induction of acute lung inflammation, C57/B16 mice were divided into 3 groups, slightly anesthetized with pentobarbital and physiological saline ( $\mathrm{n}=4)$, PAF $(2188 \mu \mathrm{g} / \mathrm{ml}$ or $5470 \mu \mathrm{g} / \mathrm{kg} ; \mathrm{n}=4$ ), or lipopolysaccharide (LPS, Escherichia coli serotype 055:B5; SigmaAldrich; $250 \mu \mathrm{g} / \mathrm{kg} ; \mathrm{n}=7 \mathrm{ZZZ}$ ) was instilled intratracheally in a total volume of $50 \mu \mathrm{l}$ physiological saline. This dose was based on previous studies (19). Animals recovered quickly from the procedure with only mild discomfort. ZZZ

\section{Histological examination}

The weight of the removed lungs and livers were measured. Then tissue samples were fixed in $8 \%$ buffered formalin $(24 \mathrm{~h})$ and embedded in paraffin wax (Shandon Pathcenter, Thermo-Shandon, USA). $4 \mu \mathrm{m}$ sections were cut and Haematoxylin-Eosin (H\&E) staining was performed. The samples were investigated with a LEICA DM 2500 microscope equipped with a LEICA DFC 420 camera (Leica Microsystems, Germany) and the images were acquired using LEICA Application Suite (LAS) V3 software.

Sample preparation for mass spectrometry 
PAF treated and control animals were sacrificed and the lungs were immediately frozen in liquid nitrogen and kept on $-70^{\circ} \mathrm{C}$ until analysis. The frozen lung tissue was homogenized with homogenizer chilled in liquid nitrogen and the homogenate was dissolved in ice-cold lysis buffer $(50 \mathrm{mM}$ Tris, $1 \mathrm{mM}$ EDTA, $17 \mathrm{mM}$ beta-mercaptoethanol, $0.5 \%$ Triton-X100), centrifuged at maximum speed (ZZZ) at $4^{\circ} \mathrm{C}$ and the supernatant was collected. The proteins from the homogenate were precipitated with 6 volume (ZZZ) of cold acetone overnight at $-20^{\circ} \mathrm{C}$ then centrifuged (ZZZ) and the supernatant was discarded. The pellet containing the proteins was dried and re-dissolved in $25 \mathrm{mM}$ ammonium bicarbonate. For protein concentration measurement the Bradford method was utilized (ZZZ). For trypsin digestion $100 \mu \mathrm{l}$ sample and $0.25 \mathrm{ng}$ trypsin was used. The digestion was carried out at $37^{\circ} \mathrm{C}$ overnight than the reaction was stopped by the addition of $1 \mu 1$ formic acid and lyophilized. For mass spectrometry measurements the proteins were re-dissolved in $0.1 \%$ formic acid and $10 \mu \mathrm{g}$ protein was used for each analysis.

Determination of relative PAF amounts in lung samples by mass spectrometry

MRM/SRM method (ZZZ) was developed for PAF measurement. After several rounds of experimental design and optimization the following MRM transitions were found to be specific for PAF: 1041.4/1038.6, 1041.4/649.5, 1249.1/1222.7 and 1249.1/1245.9. Applying these MRM transitions, PAF concentration was measured using positive mode MRM scans performed on a 4000 QTRAP (ABSciex, ZZZ) mass spectrometer using NanoSpray II MicroIon Source and controlled by the Analyst 1.4.2 software (ABSciex). The spray voltage was $2800 \mathrm{~V}$, the nebulizig gas was 50 , the curtain gas was 10 , the source temperature was $70^{\circ} \mathrm{C}$ and the de-clustering potential was $130 \mathrm{~V}$. The liquid chromatography was done on an EasynLC II nano HPLC (Bruker, ZZZ) and the peptide mixture was separated on Zorbax 300SB-C18 (5x0.3 mm, $5 \mu \mathrm{m}$ pores size) column. $30 \mathrm{~min}$ acetonitrile/water gradient was used 
for separation including the following steps: $0 \%$ to $100 \%$ acetonitrile during 10 minutes, $100 \%$ acetonitrile for 5 minutes and $100 \%$ to $0 \%$ acetonitrile during 5 minutes. The flow rate was $300 \mathrm{nl} / \mathrm{min}$ during the analyses. For the determination of PAF concentration the area under the curve of the acquired spectra was calculated.

Irritant dermatitis

The irritant dermatitis was evoked as described previously (1) with minor modifications. Namely, stock solutions of PAF and phorbol-12-myristate-13-acetate (PMA) were prepared in saline and DMSO, respectively and then both compounds were further diluted in hydrophilic cream (Unguentum hydrophylicum non-ionicum, Pharmacopoeia Hungarica VIII). Animals, 12 female C57/BL6 mice, were randomized into three groups (control, PMA, and PAF) and the thickness of their ears was measured. Mice were treated by smearing $4 \times 10 \mu \mathrm{L}$ of vehicle (saline, $0.9 \% \mathrm{NaCl} Z Z Z)$, PMA $(500 \mu \mathrm{g} / \mathrm{ml})$, or PAF $(100 \mu \mathrm{g} / \mathrm{ml})$ onto the inner and outer surface of both ears. After 24 hours, thickness of the ears was measured again and tissue samples were taken for histology.

Positron emission tomography (PET)

The glucose analog ${ }^{18}$ FDG (2-[18F]fluoro-2-deoxy-D-glucose) was synthesized and labeled with the positron-decaying isotope ${ }^{18} \mathrm{~F}$ according to a previously described method (5). Images were collected using the MiniPET-II system (http://www.minipetct.hu), a dedicated small animal PET scanner (9). The normalized and random corrected list mode data was to $0.27 \times 0.27 \times 1.37 \mathrm{~mm}$ pixel sized images.

FDG-PET experiments were carried out on mice randomized into three groups (control, $\mathrm{n}=4$; PAF, $\mathrm{n}=4$; LPS, $\mathrm{n}=6$ ). The animals were fasted for a minimum of 6 hours before undergoing PET. After an injection of 4-6 MBq of ${ }^{18}$ FDG in $150 \mu$ l of phosphate buffered saline (PBS) via the tail vein, a dynamic PET scan was acquired using the small animal PET 
camera. Mice were maintained under isoflurane anesthesia during the injection and scanning periods. They were kept warm while under anesthesia to maintain a body temperature at $35^{\circ} \mathrm{C}$. Three-dimensional regions of interest (ROI) were manually drawn around the edge of the lung activity by visual inspection using BrainCad software (http://www.minipetct.hu). The standardized uptake value (SUV) was calculated by multiplying the mean concentration activity in the ROI with mouse weight and dividing by injected dose.

Blood collection and cell counting

Mice were slightly anesthetized with pentobarbital and $200 \mu \mathrm{l}$ blood was collected into a cup containing $40 \mu \mathrm{L}$ acid citrate dextrose $(\mathrm{ACD}$, Vacutainer tube Becton Dickinson Diagnostics-Preanalytical Systems Plymouth, UK) by puncture of the retrobulbar venous plexus with a $1.5 \mathrm{~mm}$ diameter glass capillary. ACD anticoagulated whole blood was analyzed by Siemens Advia-120 hematology analyzer with Multi Species software (Deerfield, IL, USA), counting the number of the white blood cells and the neutrophil granulocytes (15). In addition, the level of liver enzymes and the ions in the plasma were measured to further explore the possible toxic effects of PAF.

\section{Animal care}

Animal experiments conformed to the guidelines of the European Community (86/609/EEC). The experimental protocol was approved by the institutional Animal Care Committee of University of Debrecen.

\section{Chemicals and statistical analysis}

Chemicals, unless otherwise stated, were purchased from Sigma (St. Louis, USA) and were of analytical grade. Averages were expressed as meanststandard error (SE) of the mean. 
Significance of the differences between control and treated animals was assessed using one way analysis of variance (ANOVA) and all pair wise multiple comparison procedures (Student-Newman-Keuls Method). 


\section{Results}

PAF was tested in vivo in several doses (0.1-54.55 $\mu \mathrm{g})$ either for a short (2 weeks) or for a prolonged period (5-8 weeks) on mice. In case of the former PAF was applied daily while in case of the latter it was administered once a week. Since the most probable application of PAF in human therapy would be to treat aspergillosis which would require the drug to be introduced via the airways, presumably using inhalators, we attempted to simulate this condition by introducing PAF to mice via the airways. The drug was thus introduced after anesthesia through the nose while the applicability of the method, that is whether the liquid thus applied actually reached the alveoli, was tested in independent experiments (see Supplementary Figure). During the course of the experiments we did not observe the death of an animal due to the drug application even in the highest concentration applied. Furthermore, the weight and physical activity of the animals remained unchanged during the study period.

To test for possible morphological alterations due to the application of PAF, histological examination of the lungs, nose, and liver of the animals were carried out. As shown in Figure 1 we did not observe any signs of pathological alterations in the lungs (Figure 1A), in the nose (Figure 1B), and in the liver (Figure 1C) tissue neither following the short, nor following the prolonged application of the protein. In the highest dose $(54.55 \mu \mathrm{g})$ of PAF both the white blood cell count $\left(1.94 \pm 0.15\right.$ and $1.83 \pm 0.1810^{10} / 1$; control vs. treated, respectively; $p>Z Z Z)$ and the percentage of neutrophils remained unchanged $(2.08 \pm 0.57$ and $2.10 \pm 0.29 \%$; control $v s$. treated, respectively; p>ZZZ). Furthermore, the concentration of liver enzymes and ions in the blood did not show a significant elevation after PAF treatment (Table 1). These results clearly established that the application of PAF neither induced any gross changes in organ morphology nor did it initiate an overall immune response in the animals. 
To prove the efficiency of the PAF application - that is to clearly establish that PAF was indeed present in the lungs - $\underline{\text { mass }}$ spectrometry was used to determine the relative PAF amounts in the lungs of PAF treated mice. The relative amount of PAF was measured in control (K1-4) and PAF treated lung samples. The results demonstrate the presence of PAF in the lungs of the PAF treated animals (Figure X). - ezt még átírom a tényleges eredmények függvényében Csosz Eva.

Although, as mentioned above, the human application of PAF would most likely be through inhalation, it cannot be excluded that the drug comes in contact with the skin, or surface application will be need as well. To test for the possibility that PAF could induce local, contact inflammation, a contact dermatitis model was used where the swelling of the ears was measured. PAF treatment did not cause the swelling of the ears in the dose used. On the other hand, PMA, used as a positive control, induced a clear edema (Figure 2A), indicating inflammation. This latter observation underlined that the model was sensitive enough to detect any inflammation. In particular, ears of mice in the PMA group $(n=4)$ were markedly thicker due to edema formation $(57.5 \pm 29.2 \mu \mathrm{m})$ than their control counterparts. In contrast, PAF did not induce any inflammation, the thickness of the ears was almost identical to the control $(22.5 \pm 5.0$ and $23.8 \pm 9.2 \mu \mathrm{m}$; in the control and in the PAF treated group, respectively, $\mathrm{n}=4$ for both groups; Figure 2B).

To test the transient effects of PAF application on the lungs we developed a new noninvasive method. FDG-PET experiments were carried out on 14 mice, randomized into 3 groups. The 3D image of the body was reconstructed from the acquired images and the lungs were identified as Regions of Interest (ROI; see Figure 3 left panel). The intensity of ${ }^{18}$ FDG accumulation within the ROI was assessed as a measure of inflammation. This method enabled us to give an estimate of and to follow any occurring inflammatory reactions in individual mice. 
Figure 3 presents reconstructed images from mice that were treated with physiological saline (Figure 3A), saline containing PAF $(2188 \mu \mathrm{g} / \mathrm{ml}$; Figure 3B) or saline containing LPS (100 $\mu \mathrm{g} / \mathrm{ml}$, Figure 3C) as positive control. It is clearly visible from the images that while PAF itself did not alter the emission intensity in the lungs as compared to control, LPS caused a marked increase in emission, indicating the presence of inflammation. These observations were quantified by calculating the emissions in the ROI. Pooled data showed a significant decrease in PAF treated group $(0.50 \pm 0.01 \mathrm{SUV}, \mathrm{n}=4)$ compared to the control $(0.72 \pm 0.04$ SUV, $n=4)$ and a significant elevation in the LPS treated animals $(0.99 \pm 0.09$ SUV, $n=6)$. To validate the PET results, we sacrificed some animals from each group and conducted histological examination. H\&E staining of the sections confirmed the absence of any inflammation in the control and the PAF-treated, while the presence of extensive inflammation in the LPS-treated animals (Figure 3A-C right panel).

Taken together our results presented here confirmed that PAF, in the intended therapeutic concentration, has no harmful effects in vivo. Furthermore, the methods introduced here enable the non-invasive characterization and follow-up of occurring inflammation of the lungs and the efficacy of the treatment. 


\section{Discussion}

Aspergillus species have emerged as important causes of morbidity and mortality in immunocompromised patients $(18,2,12)$ The treatment of aspergillosis might include chemotherapy and surgical treatment. The primary antimycotic agents against invasive pulmonary aspergillosis are voriconazole and amphotericin-B (D-AMB) ZZZ. The lipid formulations of amphotericin-B (LFABs), itraconazole, and caspofungin are used for salvage therapy while posaconazole is commonly used for prophylaxis of invasive aspergillosis in immunosuppressed patients. In allergic bronchopulmonary aspergillosis, itraconazole and corticosteroids are recommended. Patients with aspergillomas usually need surgical resection or intracavitary antifungal therapy. Chronic cavitary pulmonary aspergillosis and chronic necrotizing pulmonary aspergillosis require long-term antifungal therapy (24). Despite the best treatment available, survival rate at 12 weeks is around $70 \%$ among voriconazole-treated and around 60\% among D-AMB-treated patients with invasive pulmonary aspergillosis (3). Since the efficacy of the antifungal therapy is not complete in any other type of aspergillosis either, the development and introduction of new antimycotic agents into human therapy would be beneficial beyond doubt.

ZZZ ide PAF hatása a gombákra. Koncentráció és a koncentráció amit mi használtunk a mostani mérésekben. The Penicillium-derived anifungal protein has been shown to be very effective to inhibit the growth of most zoo-pathogen fungi in vitro (ZZZ). The effects of PAF include ZZZ (ZZZ). These effects were achieved at a concentration of ZZZ. The concentrations used in these experiments were thus determined on the basis of our earlier experiments (10), where membrane hyperpolarization and obvious signs of apoptosis in Aspergillus hyphae or protoplasts were observed after treatment with PAF. In our study we, therefore, used PAF concentrations ZZZ 10-50 $\mu \mathrm{g} / \mathrm{ml}$.

ZZZ Ide a mass spectr data hogy PAF tenylegesen jelen volt a tudoben. 
It is important to stress that mice treated with PAF showed no sign of any change in their behavior. They maintained regular activity as assessed by their normal water and food intake as well as the amount of spontaneous exercise they did (distance and speed of running in an activity wheel; data not shown ZZZ). These are clearly in line with our earlier in vitro studies where we found no toxic effects of PAF on mammalian cells, on neurons and on skeletal muscle fibers in particular (22). In those studies a wide variety of cells - including neurons, astrocytes, and skeletal muscle fibers - were examined to explore whether PAF has any acute effect on these cells similar to those described on fungal cells. The hyperpolarization-activated nonspecific cationic current and the depolarization-activated current of bushy cells from the ventral cochlear nucleus were not altered significantly. The activation thresholds and the amplitudes of depolarization activated $\mathrm{K}^{+}$currents of hippocampal neurons were not affected by PAF. No effects were observed on astrocytes either. PAF also failed to alter significantly the current of the delayed rectifier potassium channel and L-type $\mathrm{Ca}^{2+}$ currents of the skeletal muscle fibers. Taken together, our present observation with in vivo application of PAF clearly suggests that this antifungal drug - in the concentration range used - has no effect on the spontaneous activity of the treated animals.

Several organs, including the lungs, the liver, and the kidneys, taken from the group treated for 2 weeks once a day or for 8 weeks treated once a week underwent histological examination. The organs were always taken from the animals that received the highest ZZZ concentration of PAF. The fact that no morphological change was observed in these experiments strongly argues that PAF has no ZZZ. This observation, in case of the liver, the kidneys, and the skeletal muscles, is substantiated by the finding that liver enzymes, the concentration of the important ions, and creatine kinase levels, respectively, in blood of PAF treated animals were essentially identical to those of control animals (Table 1). 
In earlier experiments the possible in vitro pro-inflammatory action of PAF was examined on human blood (ZZZ). In those studies blood samples were incubated with different concentrations of PAF and the levels of IL-6, IL- 8 and TNF- $\alpha$ were measured. The rise in the production of cytokines was minimal compared to the effect of LPS. Here we examined ZZZ ide blood data on WBC and neutrophil and ...

These observations are in line with the data presented here using the FDG-PET technique. ZZZ Ide, hogy a PET alkalmas erre ref ZZZ. The PAF-induced decrease in the SUV values during the PET experiments shows also positive or at least no toxic effects of the drug. Similar data were found on some antineoplastic drugs in cell cultures (11).

Earlier studies have demonstrated that PAF has inhibiting effects on several dermatophytes, which are a group of morphologically and physiologically related molds affecting the keratinous tissue of vertebrates (4). This raises the possibility that PAF could have a potential therapeutic use in dermatophytosis. Such application would inevitably require that local application of PAF on the skin should not evoke any local inflammatory response. ZZZ model ref ZZZ Our results from the irritative dermatitis experiments prove that PAF is a potential candidate for the treatment of topical and systemic dermatophytosis.

These results proved the safety of PAF, so we can soon move to experiments evaluating the efficacy of PAF as a promising antifungal therapy. 


\section{Acknowledgement}

This work was supported by grants from the Hungarian Scientific Research Found (OTKA CK 77515, K75864), from the Hungarian Ministry of Education (TÁMOP-4.2.2-08/1/20080019 and TÁMOP 4.2.1./B-09/1/KONV-2010-0007) and KMA 0149/3.0 grant from the Research 


\section{References}

1. Bai, P., C. Hegedus, E. Szabó, L. Gyüre, E. Bakondi, A. Brunyánszki, S. Gergely, C. Szabó, L. Virág. 2009. Poly(ADP-ribose) polymerase mediates inflammation in a mouse model of contact hypersensitivity. J. Invest. Dermatol. 129:234-238.

2. Denning D. W. 1998. Invasive aspergillosis. Clin. Infect. Dis. 26:781-803.

3. Denning D. W., P. Ribaud, N. Milpied, D. Caillot, R. Herbrecht, E. Thiel, A. Haas, M. Ruhnke, H. Lode. 2002. Efficacy and safety of voriconazole in the treatment of acute invasive aspergillosis. Clin. Infect. Dis. 34:563-71.

4. Galgoczy L., T. Papp, I. Pocsi, N. Hegedus, C. Vagvolgyi. 2008. In vitro activity of Penicillium chrysogenum antifungal protein (PAF) and its combination with fluconazole against different dermatophytes. Antonie Van Leeuwenhoek 94:463-470.

5. Hamacher K., H. H. Coenen, G. Stöcklin. 1986. Efficient stereospecific synthesis of no-carrier-added 2-[18F]-fluoro-2-deoxy-D-glucose using aminopolyether supported nucleophilic substitution. J. Nucl. Med. 27(2):235-238.

6. Hegedüs N., É. Leiter, B. Kovács, V. Tomori, N.-J. Kwon, T. Emri, F. Marx, G. Batta, L. Csernoch, H. Haas, J.-H. Yu, I. Pócsi. 2011. The small molecular mass antifungal protein of Penicillium chrysogenum - a mechanism of action oriented review. J. Basic Microbiol. In press.

7. Howard S. J., D. Cerar, M. J. Anderson, A. Albarrag, M. C. Fisher, A. C. Pasqualotto, M. Laverdiere, M. C. Arendrup, D. S. Perlin, D. W. Denning. 2009. Frequency and evolution of azole resistance in Aspergillus fumigatus associated with treatment failure. Emerg. Infect. Dis. 15(7):1068-1076.

8. Kaiserer L., C. Oberparleiter, R. Weiler-Gorz, W. Burgstaller, E. Leiter, F. Marx. 2003. Characterization of the Penicillium chrysogenum antifungal protein PAF. Arch. Microbiol. 180:204-210. 
9. Kis S. A., M. Emri, G. Opposits, T. Bükki, I. Valastyán, G. Hegyesi, J. Imrek, G. Kalinka, J. Molnár, D. Novák, J. Végh, A. Kerek, L. Trón, L. Balkay. 2009. Performance test of the miniPET-II small animal according to the NEMA NU4-2008 standard. IEEE Nucl. Sci. Conf. Rec. (NSS/MIC) 3185-3189.

10. Leiter E., H. Szappanos, C. Oberparleiter, L. Kaiserer, L. Csernoch, T. Pusztahelyi, T. Emri, I. Pócsi, W. Salvenmoser, F. Marx. 2005. Antifungal Protein PAF Severely Affects the Integrity of the Plasma Membrane of Aspergillus nidulans and Induces an Apoptosis-Like Phenotype. Antimicrob. Agents. Chemother. 49.6:2445-2453.

11. Marian T., L. Balkay, L. Tron , Z. T. Krasznai, J. Szabo-Peli, Z. Krasznai. 2005. Effects of miltefosine on membrane permeability and accumulation of [99mTc]hexakis-2-methoxyisobutyl isonitrile, 2-[18F]fluoro-2-deoxy-d-glucose, daunorubucin and rhodamine123 in multidrug-resistant and sensitive cells. Eur. J. Pharm. Sci. 24:495-501.

12. Marr K. A., T. Patterson, D. Denning. 2002. Aspergillosis: pathogenesis, clinical manifestations, and therapy. Infect. Dis. Clin. North. Am. 16(4):875-94. vi.

13. Marx F., H. Haas, M. Reindl, G. Stöffler, F. Lottspeich, B. Redl. 1995. Cloning, structural organization and regulation of expression of the Penicillium chrysogenum paf gene encoding an abundantly secreted protein with antifungal activity. Gene 167:167-171.

14. Marx F., U. Binder, E. Leiter, I. Pócsi. 2008. The Penicillium chrysogenum antifungal protein PAF, a promising tool for the development of new antifungal therapies and fungal cell biology studies. Cell. Mol. Life. Sci. 65:445-454. 
15. Miszti-Blasius K., I. B. Debreceni, S. Felszeghy, B. Dezső, J. Kappelmayer. 2011. Lack of P-selectin glycoprotein ligand-1 protects mice from thrombosis after collagen/epinephrine challenge. Thromb. Res. 127(3):228-234.

16. Oberparleiter C., L. Kaiserer, H. Haas, P. Ladurner, M. Andratsch, F. Marx. 2003. Active internalization of the Penicillium chrysogenum antifungal protein PAF in sensitive aspergilli. Antimicrob. Agents. Chemother. 47:3598-3601.

17. Palicz Z., M. Füzi, P. Szentesi, C. Hegedüs, E. Leiter, I. Pocsi, L. Csernoch. 2010. In vivo test of a highly stable antifungal protein (PAF) against lung aspergillosis. Acta. Physiol. Hung. 97:468.

18. Patterson T. F., W. R. Kirkpatrick, M. White, J. W. Hiemenz, J. R. Wingard, B. Dupont, M. G. Rinaldi, D. A. Stevens, J. R. Graybill. 2000. Invasive aspergillosis: disease spectrum, treatment practices, and outcomes. I3 Aspergillus Study Group. Medicine (Baltimore) 79:250-60.

19. Puljic R., E. Benediktus, C. Plater-Zyberk, P. A. Baeuerle, S. Szelenyi, K. Brune, A. Pahl. 2007. Lipopolysaccharide-induced lung inflammation is inhibited by neutralization of GM-CSF. Eur. J. Pharmacol. 557:230-235.

20. Sallam N., A. Fisher, S. Golbidi, I. Laher. 2011. Weight and inflammation are the major determinants of vascular dysfunction in the aortae of $\mathrm{db} / \mathrm{db}$ mice. NaunynSchmied. Arch. Pharmacol. 383:483-492.

21. Smith, N. L., and D. W. Denning. 2011. Underlying conditions in chronic pulmonary aspergillosis, including simple aspergilloma. Eur. Respir. J. 37:865-872.

22. Szappanos H., G. P. Szigeti, B. Pál, Z. Rusznák, G. Szűcs, É. Rajnavölgyi, J. Balla, G. Balla, E. Nagy, É. Leiter, I. Pócsi, F. Marx, L. Csernoch. 2005. The Penicillium chrysogenum-derived antifungal peptide shows no toxic effects on mammalian cells in the intended therapeutic concentration. Naunyn-Schmied. Arch. Pharmacol. 371:122-132 
23. Tillie-Leblond I., and A. B. Tonnel. 2005. Allergic bronchopulmonary aspergillosis. Allergy 60:1004-1013

24. Walsh T. J., E. J. Anaissie, D. W. Denning, R. Herbrecht, D. P. Kontoyiannis, K. A. Marr, V. A. Morrison, B. H. Segal, W. J. Steinbach, D. A. Stevens, J. A. Burik, J. R. Wingard, T. F. Patterson. 2008. Treatment of Aspergillosis: Clinical Practice, Guidelines of the Infectious Diseases Society of America. Clin. Infect. Dis. 46:327-60 Basu S., R. Kumar, A. Alavi. 2010. PET and PET/CT imaging in infection and inflammation: Its critical role in assessing complications related to therapeutic interventions in patients with cancer. Indian J. Cancer. 47:371379.

Knight S.B., D. Delbeke, J. R. Stewart, M. P. Sandler. 1996. Evaluation of pulmonary lesions with FDG-PET: Comparison of findings in patients with and without a history of prior malignancy. Chest. 109:982-988.

Walsh T. J., E. Roilides, K. Cortez, S. Kottilil, J. Bailey, C. A. Lyman. 2005. Control immunoregulation, and expression of innate pulmonary host defenses against Aspergillus fumigatus. Med. Mycol. Suppl. 1. 43:S165-S172.

(x) Soubani A. O., and P. H. Chandrasekar. 2002. The clinical spectrum of pulmonary aspergillosis. Chest 121(6):1988-99 
Figure legends

Figure 1. Histological investigation of various mouse tissues after PAF treatment

A. Haematoxylin-Eosin stained sections of lungs from control (left) and PAF treated (5 $\mu \mathrm{g}$; right) mice at two magnifications. Section of nose (B) and liver (C). There is no pathological sign of PAF treatment on these tissues.

Figure 2. Lack of inflammatory effects of PAF in an irritant dermatitis model on mouse ears Irritant dermatitis reaction was induced in mice by smearing PMA on both sides of the ears. A. Sections of ears were stained with haematoxylin and eosin. PMA-induced irritative dermatitis resulted in edema formation in the dermis. PAF-treated mice showed no signs of inflammation. (Scale bar corresponds to $50 \mu \mathrm{m}$ for the images). B. Average increase of ears' thickness. * corresponds to significant $(\mathrm{p}<0.05)$ difference from control.

Figure 3. Detection of inflammatory effects of PAF in mouse lungs by Mini PET examination FDG-PET images (left column) and H\&E stained lung sections from control (A), PAF (B) and LPS (C) treated mouse. ROIs marked by red lines on the tranzaxial PET pictures represent the place of lung where the SUV calculations have done. Note the higher intensity areas on the tranzaxial and coronal PET image (marked with arrow) and the extensive acute inflammation in the lung in the presence of LPS.

Figure X. Relative PAF amount determined by mass spectrometry.

The figure shows the PAF amounts in different lung samples. K1, K2, K3 and K4 represent the lung from control, untreated mice, P0, P1, P2, P3 and P4 represent the lung from PAF treated mice and PAF represents the positive control containing lung from untreated mice plus 500 fmol PAF. The bars represent the results for duplicate analyses. 